\title{
Optimization design for eye-in-hand robotics Based on A New Binocular Stereo Vision Servoing Model
}

\author{
Jin $\mathrm{Mei}^{1, \mathrm{a}}$, Jin $\mathrm{Ju}^{2, \mathrm{~b}}$, Hao Guangpu $\mathrm{u}^{3, \mathrm{c}}$ \\ ${ }^{1}$ Measurement Technology and Instrumentation Key lab of Hebei Province, Yanshan university, \\ Qinhuangdao 066004, China \\ ${ }^{2}$ School of Civil Engineering, Hebei University of Technology, Tianjin 300132,China \\ ${ }^{3}$ Hebei Vocational \& Technical College of Building Materials, Qinhuangdao 066004, China \\ ${ }^{a}$ meijin297@126.com, ${ }^{b}$ jinju02@126.com, ${ }^{c}$ qhdhgpi@163.com
}

Keywords: eye-in-hand; GMC; optimization controller, Binocular Stereo Vision Servoing Model

\begin{abstract}
This paper presents an attractive image-based visual servoing approach for eye-in-hand robotic systems based on new binocular stereo vision servoing model. The major contribution of this work is in devising an optimization controller to control the velocity of the camera and GMC(Generic Model Control) is introduced to confirm the output trajectory of the system. The scheme does not deed to know the geometry model of the system. By selecting proper parameters to adjust transient time, the time for the output to follow prearranged track, is adjusted. Asymptotic convergence of the image errors to zero is proved by Lyapunov theory. Simulation results are presented for 6 DOF eye-in-hand system, which clearly show the validity of the proposed approach.
\end{abstract}

\section{Introduction}

Visual servoing is an approach to control motion of robot manipulator using visual feedback signals from a vision system ${ }^{[1]}$. This has been one of the most active topics. This paper has concentrated on the image-based eye-in-hand vision servoing. Based on the new binocular stereo vision servoing model, considering the design problem of image-based vision servoing with ideal camera model under the circumstance of precise calibration, this paper designs an optimization controller. It introduces GMC to confirm the transient behavior of the system, which can make the image feature move according to the anticipative trajectory ${ }^{[2]}$. It realizes positioning control based on image error. The asymptotic convergence of image errors to zero is proved by Lyapunov theory.

\section{Preliminaries}

$F 、 F^{*}$ represent current and desired camera frames respectively. $P_{i}\left(P_{i}^{*}\right) \in \mathfrak{R}^{3}$ are the image projections of the feature point $P_{i}$ in $F\left(F^{*}\right)$ expressed in pixels coordinates. $m_{i}$ and $m_{i}^{*}$ respectively are the current and desired image projections of the feature point $P_{i}$ in image planes. $v_{c}=\left[\begin{array}{lll}v_{x} & v_{y} & v_{z}\end{array}\right]^{T}$ and $\omega_{c}=\left[\begin{array}{lll}\omega_{x} & \omega_{y} & \omega_{z}\end{array}\right]^{T}$ are translational and rotational velocities of left and right cameras respectively. To be convenient for analysis, we have some assumptions ${ }^{[3]}$.

The positioning task of binocular stereo vision servoing is to control the two cameras moving) from some initial position to desired position, and finally make $m_{i}-m_{i}^{*}=0$ or $F^{*}$ coincide with $F$ finally. The velocity relationship between the end-effector (or camera) and the object is

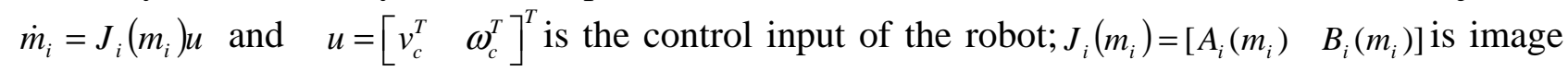
Jacobian matrix. The image Jacobian matrix of the new binocular stereo vision servoing model is 


$$
\begin{aligned}
& {\left[\begin{array}{c}
\dot{x} \\
\dot{y} \\
\dot{\theta}
\end{array}\right]=\left[\begin{array}{cccccc}
\frac{\theta-x}{B} & 0 & \frac{x(x-\theta)}{f B} & +\frac{x y}{f} & -\frac{f^{2}+x^{2}}{f} & +y \\
0 & \frac{\theta-x}{B} & \frac{y(x-\theta)}{f B} & +\frac{f^{2}+y^{2}}{f} & -\frac{x y}{f} & -x \\
\frac{\theta-x}{B} & 0 & \frac{\theta(x-\theta)}{f B} & \frac{+y \theta}{f} & -\frac{f^{2}+x \theta}{f}+y
\end{array}\right] \cdot\left[\begin{array}{c}
T_{X} \\
T_{Y} \\
T_{Z} \\
\omega_{X} \\
\omega_{Y} \\
\omega_{Z}
\end{array}\right]} \\
& A_{i}\left(m_{i}\right)=\left[\begin{array}{ccc}
\frac{\theta-x}{B} & 0 & \frac{x(x-\theta)}{f B} \\
0 & \frac{\theta-x}{B} & \frac{y(x-\theta)}{f B} \\
\frac{\theta-x}{B} & 0 & \frac{\theta(x-\theta)}{f B}
\end{array}\right], \quad B_{i}\left(m_{i}\right)=\left[\begin{array}{ccc}
+\frac{x y}{f} & -\frac{f^{2}+x^{2}}{f} & +y \\
+\frac{f^{2}+y^{2}}{f} & -\frac{x y}{f} & -x \\
+\frac{y \theta}{f} & -\frac{f^{2}+x \theta}{f} & +y
\end{array}\right]
\end{aligned}
$$

The state equation of the vision servoing system is constituted by $N$ feature points of the object. $\dot{m}=J(m) u=\left[\begin{array}{ll}A(m) & B(m)\end{array}\right] u$

where $\quad m=\left[\begin{array}{llll}m_{1}^{T} & m_{2}^{T} & \cdots & m_{N}^{T}\end{array}\right]^{T} \quad J(m, Z)=\left[\begin{array}{lllll}J_{1}^{T}\left(m_{1}, Z_{1}\right) & J_{2}^{T}\left(m_{2}, Z_{2}\right) & \cdots & J_{N}^{T}\left(m_{N}, Z_{N}\right)\end{array}\right]^{T}$

$$
A(m)=\left[\begin{array}{lllll}
A_{1}^{T}\left(m_{1}\right) & A_{2}^{T}\left(m_{2}\right) & \cdots & A_{N}^{T}\left(m_{N}\right)
\end{array}\right]^{T} \quad B(m)=\left[\begin{array}{llll}
B_{1}^{T}\left(m_{1}\right) & B_{2}^{T}\left(m_{2}\right) & \cdots & B_{N}^{T}\left(m_{N}\right)
\end{array}\right]^{T}
$$

We introduce the output equation $y=m$; where $y=\left[\begin{array}{llll}y_{1}^{T} & y_{2}^{T} & \cdots & y_{N}^{T}\end{array}\right] \in \mathfrak{R}^{3 N}$. State equation of the entire system is written as $\left\{\begin{array}{c}\dot{m}=[A(m) \quad B(m)] u \\ y=m\end{array}\right.$

The derivation of output equation is: $\dot{y}=\dot{m}=[A(m) \quad B(m)] u=A(m) v_{c}+B(m) \omega_{c}$

\section{Design and Stability Analysis of the Controller}

We introduce GMC to confirm the output trajectory according to the anticipated trajectory from an initial state to terminative state ${ }^{[4]}$. Considering the following differential equation to determine the state trajectory

$$
\dot{y}_{r}=k\left[y^{*}-y_{r}\right]
$$

Where $y_{r}$ and $y^{*}$ are reference trajectory and desired value of the feature points, and $k$ is the operator. We choose operator in the form of proportional differentiator

$$
k[\varepsilon]=K_{1} \varepsilon+K_{0} \frac{d \varepsilon}{d t}
$$

where $K_{1}=\operatorname{diag}\left\{K_{11}, K_{11}, K_{12}, K_{12} \cdots K_{1 N}, K_{1 N}\right\} \quad K_{0}=\operatorname{diag}\left\{K_{01}, K_{01}, K_{02}, K_{02} \cdots K_{0 N}, K_{0 N}\right\}, K_{1}$ and $K_{0}$ are positive definite diagonal matrix, besides the elements in the diagonal of $K_{0}$ are same. Then

$$
\dot{y}_{r}=K_{1}\left(y^{*}-y_{r}\right)+K_{0} \frac{d\left(y^{*}-y_{r}\right)}{d t}
$$

$y_{r}(0)=y_{0}$ is the initial value. We design $u$ to make the output track the anticipated trajectory as much as possible. Assuming $y=y_{r}\left(y(0)=y_{r}(0)\right)$, the following equation is obtained

$$
\min _{u} \int_{0}^{t_{f}}\left[a(x, u)^{T} W a(x, u)\right] \quad \text { s.t. } \dot{m}=[\theta A(m) \quad B(m)] u \quad|u| \leq \beta
$$

where $\quad a(x, u)=\dot{y}-\dot{y}_{r}=[\theta A(m) \quad B(m)] u-K_{1}\left(m^{*}-m\right)-K_{0} \frac{d\left(m^{*}-m\right)}{d t}$

$W$ is a positive definite weighted matrix. The value of $\beta$ is determinated by the maximum speed of the actual control system. The above solution problem of the optimization is converted into:

$[\theta A(m) \quad B(m)] u-K_{1}\left(m^{*}-m\right)-K_{0} \frac{d\left(m^{*}-m\right)}{d t}=0 \quad \theta A(m) v_{c}+B(m) \omega_{c}-K_{1}\left(m^{*}-m\right)-K_{0} \frac{d\left(m^{*}-m\right)}{d t}=0$

Rearranging the above equations by replacing the estimated value $\hat{\theta}$ by $\theta$, we obtained two equivalent controller expressions containing translational and rotational velocity of the camera, 


$$
\begin{aligned}
& \hat{\theta} A(m) \nu_{c}=\varepsilon K_{1}\left(m^{*}-m\right)+K_{0} \frac{d\left(m^{*}-m\right)}{d t} \\
& B(m) \omega_{c}=(1-\lambda) K_{1}\left(m^{*}-m\right) \\
& \text { where } 1>\lambda>0 \text {, then } \\
& v_{c}=\operatorname{pinv}(A(m)) \hat{\theta}^{-1}\left(\lambda K_{1}\left(m^{*}-m\right)+K_{0} \frac{d\left(m^{*}-m\right)}{d t}\right) \\
& \omega_{c}=\operatorname{pinv}(B(m))(1-\lambda) K_{1}\left(m^{*}-m\right)
\end{aligned}
$$

Where $\operatorname{pinv}(A(m))$ and $\operatorname{pinv}(B(m))$ are the pseudo-inverse matrixs of $A(m)$ and $B(m)$.

(10) and (11) are the control input commands of the robot. The asymptotic convergence of image errors to zero is proved by Lyapunov theory.

Theorem 1 For a given system (2) and control (10) and (11), there exists a smooth function of storage $V(m)=\frac{1}{2}\left(m-m^{*}\right)^{T}\left(m-m^{*}\right)$, the system is globally asymptotically stable.

Proof: The differential of the storage function is as follows:

$$
\begin{aligned}
& \dot{V}(m)=\left(m^{*}-m\right)^{T}\left(\dot{m}^{*}-\dot{m}\right)=-\left(m^{*}-m\right)^{T} \dot{m} \\
& =-\left(m^{*}-m\right)^{T}\left[\begin{array}{ll}
A(m) & B(m)
\end{array}\right]\left[\begin{array}{c}
v_{c} \\
\omega_{c}
\end{array}\right]=-\left(m^{*}-m\right)^{T}\left[A(m) V_{c}+B(m) \omega_{c}\right] \\
& \left.=-\left(m^{*}-m\right)^{T}\left(\lambda K_{1}\left(m^{*}-m\right)+\mu K_{0} \frac{d\left(m^{*}-m\right)}{d t}+(1-\lambda) K_{1}\left(m^{*}-m\right)+(1-\mu) K_{0} \frac{d\left(m^{*}-m\right)}{d t}\right)\right) \\
& =-\left(m^{*}-m\right)^{T} K_{1}\left(m^{*}-m\right)-\left(m^{*}-m\right)^{T} K_{0} \frac{d\left(m^{*}-m\right)}{d t}=V_{1}+V_{2} \\
& \text { where } \quad V_{1}=-\left(m^{*}-m\right)^{T} K_{1}\left(m^{*}-m\right) \quad V_{2}=-\left(m^{*}-m\right)^{T} K_{0} \frac{d\left(m^{*}-m\right)}{d t}
\end{aligned}
$$

It is easy to know that $V_{1}$ is negative definite. The second term $V_{2}$ is $-e^{T} K_{0} \dot{e}$, thus : $\dot{V}(m)=e^{T} \dot{e}=V_{1}-e^{T} K_{0} \dot{e}$.Take the second term to the left of the equation,then we have $e^{T}\left(I+K_{0}\right) \dot{e}=V_{1}$. Because $V_{1}$ is negative definite, the left of the equation is also negative definite. Since the elements in the diagonal of $K_{0}$ are same, $K_{0}$ can also be written as $\gamma \cdot I \cdot \gamma$ is an adjustable positive coefficient, so the left of the equation becomes $\gamma e^{T} \dot{e} \cdot e^{T} \dot{e}$ is negative definite, that is, $\dot{V}(m) \leq 0$. So, when $u=0$ and $y=m^{*}, m \rightarrow m^{*}$. Thus it is known that the desired position of the system is detectable. Then according to LaSalle invariant set theorem, the asymptotic stability of the system desired point $m^{*}$ is proved.

Substitute the controllers (10) and (11) into system (2), we know that the closed loop system is first-order and index-attenuating. By adjusting the elements in the coefficient matrixes of $K_{1} 、 K_{0}$, adjustion time or transient time can be adjusted, which can achieve the aim of adjusting the movement process of the cameras. Thus the cameras can reach the desired position from the arbitrary initial position according to the predefined transition process. The proof is completed.

\section{Simulation}

Here we use Robotics Toolbox for Matlab and Visual Servoing to simulate ${ }^{[5]}$. The controlled object is puma-560 robot without considering robot friction torque. Choose its four points as feature points. The origin coordinates and the corresponding desired coordinates of the image feature points respectively are

$$
m=\left[\begin{array}{l}
x \\
y \\
\theta
\end{array}\right]=\left[\begin{array}{cccc}
302.2 & 211.4 & 306.4 & 407.4 \\
160.2 & 290 & 364 & 217.7 \\
208.2 & 121.4 & 207.4 & 307.4
\end{array}\right] \quad m^{*}=\left[\begin{array}{l}
x \\
y \\
\theta
\end{array}\right]=\left[\begin{array}{cccc}
211 & 211 & 481 & 481 \\
131 & 381 & 381 & 131 \\
81 & 81 & 311 & 311
\end{array}\right]
$$

The focus of left and right cameras is $0.016 \mathrm{~m}$. The resolutions of $x$ axes and $y$ axes of the image plane are both 80000pixels/m. $B=0.1 m, K_{0}=K_{1}=0.25 \operatorname{diag}\{1,2, \cdots 1,1\} \in \mathfrak{R}^{12},{ }_{\lambda}=\mu=\frac{1}{12}$. 
The above half of Fig.1 is the error curve in left image points coordinates, and the down half of Fig. 2 is the error curve in right image points coordinates. The above and down half of Fig.3 are translational and rotational velocities of the left and right camera respectively. Fig.3 are trajectories in the left and right images of the target points respectively. From the simulation results, after 30s'adjustment, the error of image feature points basically tends to be zero, and the control effect is good. Therefore, the proposed method can drive the camera moving from any initial position to the desired position stably with faster dynamic adjustment process and smaller positioning error.
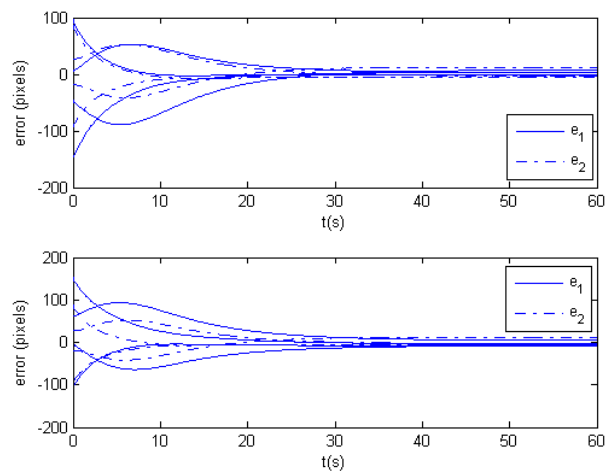
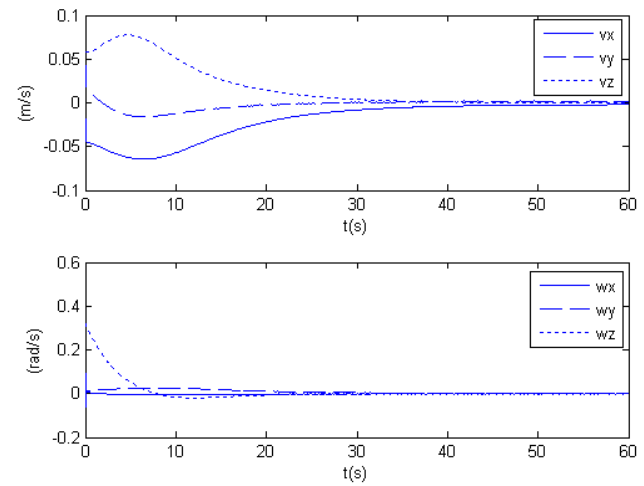

Fig.1 Errors in image points coordinates Fig.2 Translational and rotational velocities of the camera
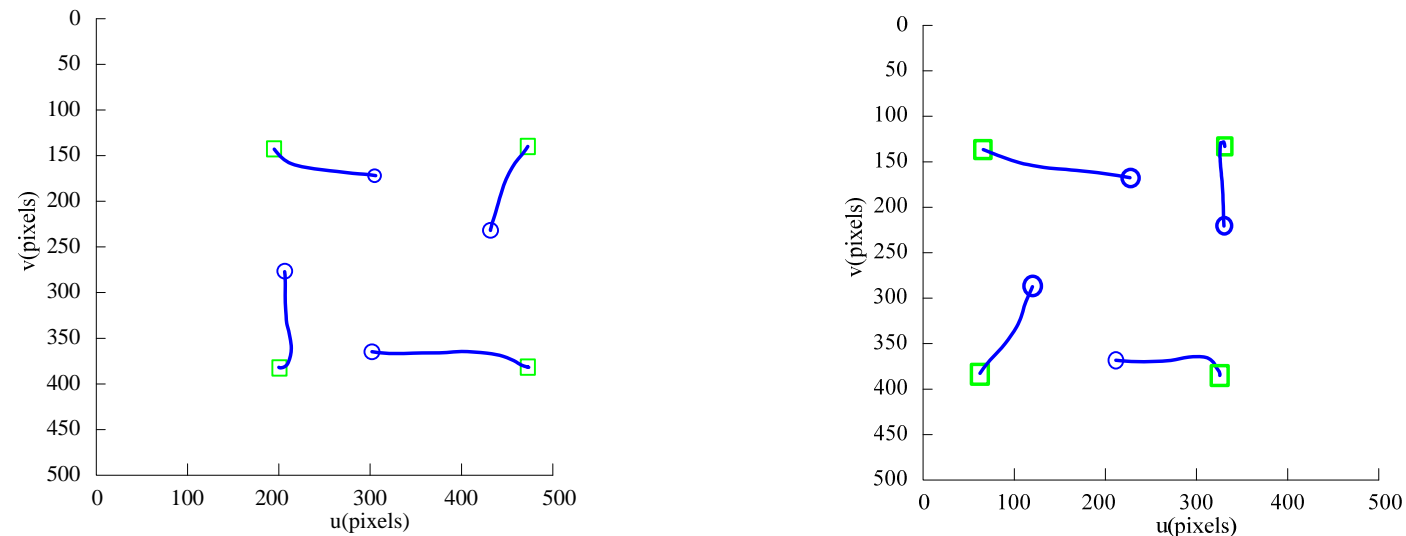

Fig.3 Trajectories in the image of the target points(Initial location: circle , desired: block)

\section{Summary}

Based on the new binocular stereo vision servoing model, aiming at the task of positioning, this paper designs an optimization controller. Under the circumstance of known intrinsic and extrinsic parameters of the left and right cameras, GMC and optimization control are used to design the visual servoing controller and gives translation and rotation velocities of the two cameras, and finally realizes the positioning task. The system is globally asymptotically stable proved by the Lyapunov theory. Simulation results show its validity.

\section{References}

[1] Saeed B. Niku. Introduction to Robotics Analysis, System, Applications. Beijing: Publishing House of Electronics Industry,2004:3-5

[2] L. Lee. Nonlinear Process Control: Application of Generic Model Control. London, Springer Verlay,1993

[3] Huiguang Li, Mei Jin, and Liying Zou. A New Binocular Stereo Visual Servoing Model.[J] IEEE Pacific-Asia Workshop on Computational Intelligence and Industrial Application,2008, vol.1: 461-465.

[4] B. A. Lundberg, L. W. Bezanson. Enhanced Robustness of Generic Model Control Using Derivative Feedback. AIChEJ, 37(11):1634-1644

[5] P.I.Corke. Robotics Toolbox for MATLAB. http://www.cat.csiro.au/cmst/staff/pic/robot 